\title{
Can nasal continuous positive airway pressure be used as primary respiratory support for infants with meconium aspiration syndrome?
}

\author{
Kelsey A. Montgomery ${ }^{1} \cdot$ Rebecca S. Rose ${ }^{1}$ \\ Received: 30 August 2018 / Accepted: 12 September 2018 / Published online: 19 October 2018 \\ (c) Springer Nature America, Inc. 2018
}

Manuscript citation: Pandita A, Murki S, Oleti TP, Tandur B, Kiran S, Narkhede S, et al. Effect of Nasal Continuous Positive Airway Pressure on Infants With Meconium Aspiration Syndrome: A Randomized Clinical Trial. JAMA Pediatr. 2018;172(2):161-5. PMID: 29204652

\section{Type of investigation}

Treatment.

\section{Question}

In infants ( $>35$ weeks gestation with BW $>2000$ g) born through meconium-stained amniotic fluid with respiratory distress (defined by Downe score $>4$ with $\mathrm{SpO} 2<90 \%$ on room air) does nasal continuous positive airway pressure (NCPAP) compared with 5-10 L/min hood oxygen reduce the risk of the need for mechanical ventilation (MV) in the first 7 days of life?

\section{Methods}

Design: Multicenter, open-label, randomized controlled trial.

Allocation: Parallel group at a 1:1 ratio of randomly alternated computer generated blocks of 2 or 4 , stratified by center.

Blinding: Open-label, unblinded (clinicians).

Kelsey A. Montgomery

Kelsey.Montgomery@ascension.org

1 Department of Pediatrics, Division of Neonatology, Indiana University School of Medicine, Riley Hospital for Children, Indianapolis, IN, USA
Follow-up period: Infants were followed until discharge from the hospital.

Setting: Three tertiary care neonatal intensive care units in India.

\section{Patients}

\section{Inclusion criteria}

Infants $>35$ weeks gestation, $>2000 \mathrm{~g}$ birth weight, born through meconium-stained fluid, admitted to the neonatal intensive care unit (NICU) within the first $24 \mathrm{~h}$ of life, with respiratory distress using Downe scoring (a tool for categorizing the degree of respiratory distress) [1], of $>4$ with capillary oxygen saturation $<90 \%$ on room air) and chest radiograph indicative of meconium aspiration syndrome.

\section{Exclusion criteria}

Severe asphyxia (Apgar score of $<3$ at $5 \mathrm{~min}$ and cord $\mathrm{pH}<7.00$ ), intubation at admission, pneumothorax or air leak on chest radiograph upon admission, major congenital malformations.

\section{Intervention}

Eligible neonates were randomized at study enrollment to either bubble NCPAP or hood oxygen. Arterial blood gas and chest radiograph were obtained prior to randomization. NCPAP pressure and fraction of inspired oxygen $\left(\mathrm{FiO}_{2}\right)$ were titrated to a target saturation of $90-95 \%$. Participants were weaned off NCPAP once saturations were persistently $>90 \%$, FIO2 requirement was $<25 \%$, and respiratory distress had resolved.

\section{Outcomes}

The primary outcome was the need for MV, defined by: (1) NCPAP failure ( $\mathrm{SpO} 2$ levels $<90 \%$ on NCPAP pressure of $6 \mathrm{~cm}$ of water and $100 \% \mathrm{FIO} 2$ ), (2) hood oxygen failure 
( $\mathrm{SpO} 2<90 \%$ for greater than $15 \mathrm{~min}$ on $100 \% \mathrm{FIO} 2)$, (3) persistent shock requiring inotropes $>10 \mu \mathrm{g} / \mathrm{kg} / \mathrm{min}$, (4) elevated partial pressure of carbon dioxide (greater than 60 $\mathrm{mm} \mathrm{Hg}$ ) with $\mathrm{pH}<7.20$, or (5) hemodynamic compromise with new-onset pneumothorax in the first 7 days of life. Secondary outcomes included the need for surfactant, culture positive sepsis, pulmonary hypertension, death, pneumothorax, duration of oxygen, and length of hospital stay.

\section{Statistical analysis and sample size}

Results were analyzed using intention-to-treat for all participants. A sample size of 66 infants per group was needed to achieve $80 \%$ power with an alpha error of 0.05 and detect a reduction of treatment failure from $30 \%$ (rate of failure in existing data on hood oxygen) to $10 \%$ in the NCPAP group. Study group comparisons of discrete variables were performed using logistic regression. Study group comparisons of continuous variables were performed using log transformation. For center comparisons, linear regression was performed.

\section{Patient follow-up}

Written informed consent was obtained via infant families within an hour of NICU admission. 91\% of those eligible were included in the study for randomization. The other $9 \%$ were excluded due to severe birth asphyxia $(n=9)$, poor respiratory effort requiring intubation at birth $(n=3)$, and consent refusal $(n=2) .100 \%$ of the randomized patients were included in the primary and secondary analyses.

\section{Main results}

\section{Overall cohort}

In total, 135 neonates were randomly assigned to the treatment group (67 to the NCPAP group and 68 to the standard treatment group). Mean birth weight was $2944 \mathrm{~g}$, average gestation was 38.2 weeks, $41.5 \%$ male. Baseline characteristics between groups did not differ.

\section{Primary outcome}

Treatment failure as defined by need for MV, occurred in 3\% of the NCPAP group, and $25 \%$ of the hood oxygen group (odds ratio, 0.09; 95\% confidence interval 0.02-0.43; $p=$ 0.002). Seventeen of the 19 infants who failed hood oxygen were placed on NCPAP prior to MV and 15 were ultimately placed on MV. Four out of the 19 infants who failed hood oxygen required high frequency ventilation (HFV) for failed conventional MV. None of the infants in the NCPAP group required HFV. The number needed to treat was 4.5 ; for every five infants with MAS who received NCPAP instead of hood oxygen one infant would avoid MV.

\section{Secondary outcomes}

More infants in the hood oxygen group required surfactant, developed culture positive sepsis, and had a longer duration of oxygen requirement than in the NCPAP group. One infant in the hood oxygen group died from pulmonary hemorrhage. One infant in the NCPAP group developed a pneumothorax.

\section{Study conclusion}

Initiation of early NCPAP instead of hood oxygen in neonates with respiratory distress due to MAS decreases the risk of subsequent MV in the first 7 days of life. For every five infants with respiratory distress due to MAS supported with NCPAP, one infant will be protected from subsequent MV.

\section{Commentary}

Meconium aspiration syndrome (MAS) continues to be a significant source of respiratory failure in the neonatal population [2, 3]. While mortality rates of MAS have decreased over the years, significant morbidity/mortality still exist, particularly from the need for MV [4]. In fact, up to $50 \%$ of neonates with MAS will need ventilator support [4] with associated costs of prolonged hospital stay, need for continued critical care, and other morbidities [5]. In neonates requiring ventilation, short-term risks include air leak syndromes and pulmonary hemorrhage, while long-term risks include prolonged respiratory compromise post-discharge, cerebral palsy and global developmental delay [5, 6].

While a large number of neonates with MAS will need respiratory support, the ideal ventilatory strategy remains unknown [7]. Since lung mechanics are altered with significant atelectasis and obstruction in MAS, respiratory management has historically included hood oxygen without positive pressure to limit the risks of worsened air trapping and resultant air leaks [7]. However, persistent high oxygen exposure is not benign and has been shown to worsen pulmonary arterial constriction in lambs as well as lead to increased neonatal risks of bronchopulmonary dysplasia, retinopathy of prematurity, and necrotizing enterocolitis $[8,9]$. As an alternative, NCPAP has been proposed for the prevention of $\mathrm{MV}$ as it provides positive pressure with reduced oxygen administration $[4,7]$. Known benefits of NCPAP include improved atelectasis, reduced air trapping, and decreased ventilation-perfusion mismatch for improved 
gas exchange, while slightly increasing the risk of pneumothorax compared to other modalities [4, 7].

The NCPAP for MAS trial evaluated the feasibility of using early NCPAP for non-invasive respiratory support in the first week of life. Strengths of this study include a randomized, multisite design with mostly inborn infants, exclusion of infants with hypoxic respiratory failure, and sufficient power to detect differences between groups. The $3 \%$ failure rate in neonates randomized to the NCPAP treatment group vs. $25 \%$ in the hood oxygen group was greater than that predicted by chance alone (odds ratio, $0.09 ; 95 \%$ CI, $0.02-0.43 ; p=0.002$ ).

Although the study was powered to show a difference in rates of treatment failure, the resource-poor setting may have generated some generalizability limitations. For instance, the Downes [1] score has good reproducibility across multiple settings, but it is not routinely used in neonatal intensive care units in the US. Additionally, while inhaled nitric oxide is considered standard therapy in the US for severe pulmonary hypertension, it was not used in this cohort which may suggest either a differing patient population or a resourcelimited setting. Likewise, given limited resource availability of higher-level support, specific treatment failure criteria may have been more generous. Finally, the potential for variability both in the clinician discretion to place neonates on either NCPAP or MV following hood oxygen failure and specific protocol differences on comorbid condition management may have been partially due to resource restrictions.

This was a well-designed and implemented study with low to moderate risk of bias that provides a good argument for the use of early NCPAP support in MAS. The study provides an intervention with relative applicability for most neonatal intensive care units in the US. The intervention of NCPAP is feasible, with relatively easy accessibility and low cost. Overall, the study presents a compelling argument for trialing early NCPAP in near-term and term infants with respiratory failure to prevent MV in the first seven days of life.

\section{EBM lesson: permuted blocked randomization}

One of the most important components in a clinical trial is randomization because it reduces sample bias-a participant's or investigator's ability to pre-emptively enroll into a desired treatment group. As such, it allows a relatively non-biased valid outcome assessment by optimizing the distribution of both known and unknown participant characteristics and prognostic variables between groups. One specific type of allocation randomization, "permuted blocked randomization", ensures participants are assigned to either treatment or control groups based on a constant predetermined ratio of allocation, such as 1:1 in this study, and alternating between a permutated block size of 2:4 patients [10,11]. In this case, two patients are enrolled into alternating groups followed by the next four patients who are enrolled in variable order into alternating groups. The process is repeated until the total enrollment is achieved, ensuring an equal number of subjects in each group when enrollment is complete. Investigators use permutation of block size to make it more difficult to predict the block size and thus, predict or influence patient group. By randomly assigning new participants in block groups with equal probability, blocked randomization helps limit selection bias, avoid group imbalance, and maintain a valid assessment [10, 11].

\section{Compliance with ethical standards}

Conflict of interest The authors declare that they have no conflict of interest.

\section{References}

1. Wood DW, Downes JJ, Leeks HI. A clinical scoring system for the diagnosis of respiratory failure: preliminary report on childhood status asthmaticus. Am J Dis Child. 1972;123:227-8.

2. Singh B, Clark R, Powers R, Spitzer A. Meconium aspiration syndrome remains a significant problem in the NICU: outcomes and treatment patterns in term neonates admitted for intensive care during a ten-year period. J Perinatol. 2009;29:497.

3. Fischer C, Rybakowski C, Ferdynus C, Sagot P, Gouyon JB. A population-based study of meconium aspiration syndrome in neonates born between 37 and 43 weeks of gestation. International journal of pediatrics. Int J Pediatr. 2012;2012:321545.

4. Bhagwat P, Murki S, Mehta A, Oleti T, Gannavaram D. Continuous positive airway pressure in meconium aspiration syndrome: an observational study. J Clin Neonatol. 2015;4:96.

5. Dargaville PA. Respiratory support in meconium aspiration syndrome: a practical guide. International journal of pediatrics. 2012; 2012:965159.

6. Anantharaj A, Bhat BV. Outcome of neonates requiring assisted ventilation. Turk J Pediatr. 2011;53:547.

7. Goldsmith JP. Continuous positive airway pressure and conventional mechanical ventilation in the treatment of meconium aspiration syndrome. J Perinatol. 2008;28(Suppl 3):S49-55.

8. Lakshminrusimha S, Russell JA, Steinhorn RH, Ryan RM, Gugino SF, Morin FC, et al. Pulmonary arterial contractility in neonatal lambs increases with 100\% oxygen resuscitation. Pediatr Res. 2006;59:137.

9. SAUGSTAD OD. Oxygen toxicity in the neonatal period. Acta Paediatr. 1990;79:881-92.

10. Broglio K. Randomization in clinical trials: permuted blocks and stratification. JAMA. 2018;319:2223-4.

11. Kernan WN, Viscoli CM, Makuch RW, Brass LM, Horwitz RI. Stratified randomization for clinical trials. J Clin Epidemiol. 1999;52:19-26. 